\title{
Heart Rate Variability Analysis during Muscle Fatigue due to prolonged Isometric Contraction
}

\author{
Andrea Guidi, Alberto Greco*, Federica Felici, Andrea Leo, Emiliano Ricciardi, Matteo Bianchi, \\ Antonio Bicchi, and Gaetano Valenza ${ }^{\ddagger}$, Enzo Pasquale Scilingo ${ }^{\ddagger *}$
}

\begin{abstract}
Fatigue can be defined as the muscular condition occurring before the inability to perform a task. It can be assessed through the evaluation of the median and mean frequency of the spectrum of the surface electromyography series. Previous studies investigated the relationship between heartbeat dynamics and muscular activity. However, exploitation of such cardiovascular measures to automatically identify muscle fatigue during fatiguing exercises is still missing. To this extent, HRV signals were gathered from 32 subjects during an isometric contraction task, and features defined in the time, frequency and nonlinear domains were investigated. We used surface electromyography to label the occurrence of muscle fatigue. Statistically significant differences were observed by comparing features related to fatigued subjects with the nonfatigued ones. Moreover, a pattern recognition system capable to achieve an average accuracy of $78.24 \%$ was implemented. These results confirmed the hypothesis that a relationship between heartbeat dynamics and muscle fatigue might exist.
\end{abstract}

\section{INTRODUCTION}

Fatigue is usually mechanically associated with the inability to further perform a task or sustain an effort, or to the inability to reach the same initial level of maximal voluntary contraction (MVC) force [1]. From an engineering point of view, the muscle fatigue process can be thought as the muscular condition occurring before the inability to perform a task [1]. Such a process can be fast or slow, but it leads to the "myoelectric manifestations of muscle fatigue" [1]. Fatigue can affect many potential sites in the neuromuscular system: the motor cortex, the excitatory drive, the control strategies of the spinal (upper) and the $\alpha$ (lower) motoneurons, the motoneuron conduction properties, the neuromuscular transmission, the sarcolemmal excitability and conduction properties, the excitation-contraction coupling, the metabolic energy supply, and the contraction mechanisms. According to the site, it is possible to define central fatigue or fatigue of the neuromuscular junction, or muscle fatigue [1]. All of them are directly or indirectly able to influence the electromyographic signal (EMG) in a very complex way. More specifically, a progressive slowing of

The research leading to these results has received partial funding from the EU H2020 project "SoftPro: Synergy-based Open-source Foundations and Technologies for Prosthetics and RehabilitatiOn" (H2020-ICT-688857).

A.G., A.G., M.B., G.V., E.P.S. are with the department of Information Engineering and Research Center "E. Piaggio", School of Engineering, University of Pisa, Via G. Caruso 16 - 56122, Pisa, Italy.

F.F. and A.B. are with the Advanced Robotics Department, Istituto Italiano di Tecnologia (IIT), Genova, Italy

A.L. and E.R. are with the IMT School for Advanced Studies Lucca, Piazza San Ponziano, 6, I-55100 Lucca, Italy

¥ Senior Authors

* Corresponding author.

e-mail: alberto.grecodcentropiaggio.unipi.it the EMG during isometric voluntary sustained contractions was observed [2]. Such a slowing has been easily described via the mean or median frequencies $(M N F$ and $M D F)$ of the power spectral density function, as suggested by [3]-[6]. For this reason, mean and median frequency are commonly considered as two meaningful frequency domain features for EMG analysis both in clinical and engineering applications. In fact, a decrease in these features is commonly used to detect fatigue in muscles using surface EMG (sEMG) signals [7].

The relation between muscular exercise and cardiovascular activity has been investigated in many previous studies. They reported an increasing heart rate (HR), and oxygen uptake [8]-[11], as well as changes in blood volume, due to a modification in left ventricular end-diastolic and end-systolic dimensions [10]. HR changes were also studied together with the EMG activity during fatiguing exercises [12], [13]. Specifically, similar rates of change in average HR and average EMG activity were reported. In addition, significant differences were observed in both EMG features, and HR in relation to different exercise durations.

Several studies have also observed significant changes in heart rate variability (HRV) spectra during aerobic exercise with different intensities or after fatiguing training sessions [14]-[16], whereas few studies have monitored heartbeat dynamics during a fatiguing task [17]-[19]. However, in all these studies, muscle fatigue was not assessed using objective measures such as those obtained by means of the EMG analysis, In addition, such parameters extracted from the analysis of the heartbeat dynamics were never used to perform an alternative automatic recognition of the muscle fatigue condition.

In sight of this, we performed an analysis of HRV series during a fatiguing exercise to evaluate differences between people with and without muscle fatigue. Moreover, the HRV features were used as input of a pattern recognition system in order to automatically detect the fatiguing condition. Such a condition was labeled by analyzing the sEMG signals during the muscular contraction.

\section{MATERIALS AND METHOD}

\section{A. Experimental protocol}

32 subjects (16 males and 16 females, $29.25 \pm 3.38$ ) were enrolled in this study. All the subjects were right-handers and did not have any physical disease at the muscular or bone system, as well as no past or current heart disease. The experimental protocol (Figure 1) consisted in three phases. In the first phase the evaluation of the maximum voluntary 


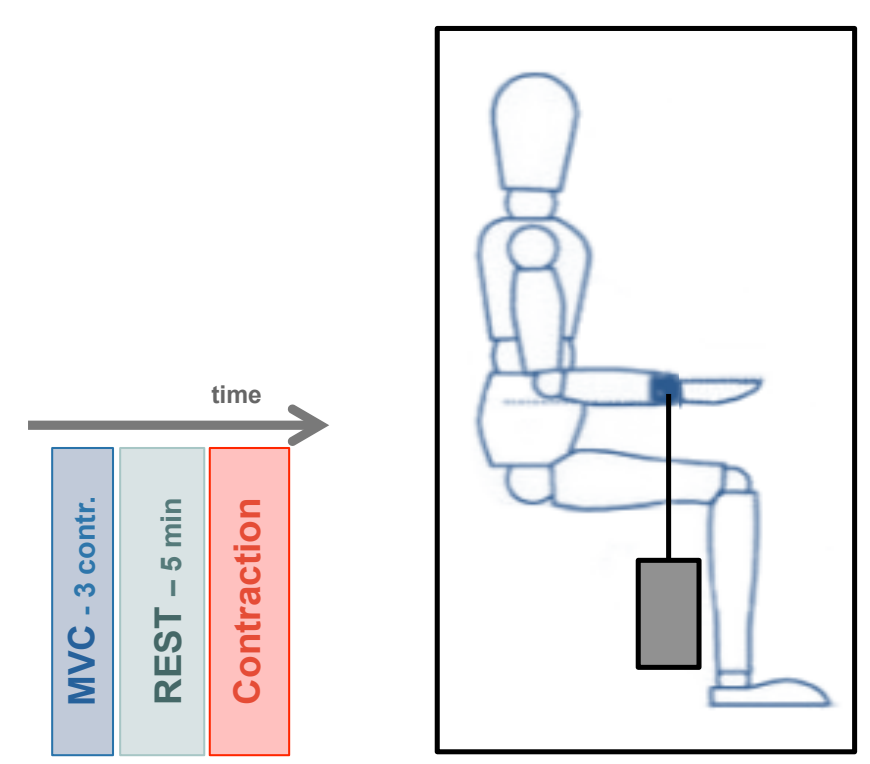

Fig. 1. Timeline of the experimental protocol (left) and position of the subject during contraction (right).

contraction (MVC) was performed by using a dynamometer and asking the subject to maximally contract the biceps three times for three seconds. MVC was determined as the mean of the three maximum forces. In the second phase, a 5 minutelong resting state was recorded. After that, in the third phase, a static contraction of the biceps brachii long head (Figure 1) was required to the subjects. A load equal to the $40 \%$ of the previously estimated MVC was used in this task [20], and the position was maintained till fatigue. The fatigue was detected when the subject was no longer able to maintain the position of the limb. During the whole experimental protocol, subjects were comfortably sat on a chair, straightening their back. A Biopac MP35 was used to acquire ECG signals, while a Bagnoli Desktop EMG Delsys was used to record sEMG signals related to the biceps brachii long head muscle. A sampling frequency equal to $2000 \mathrm{~Hz}$ was used to record both signals.

\section{B. Algorithm}

1) sEMG processing: Firstly, sEMG signals were filtered with a Infinite Impulse Response (IIR) comb notch filter (50 $\mathrm{Hz}$ ). Then a zero-phase Butterworth IIR band-pass filter was used to retain frequencies between 30 and $500 \mathrm{~Hz}$. At the end, Fourier approach was used to estimate the spectrum and thus mean (MNF) and median (MDF) frequencies within non-overlapping epochs. The Hamming window was used. Moreover, in this study, epochs lasting 1 second were chosen to estimate MNF and MDF. In fact, it was proven that in case of isometric, constant force, fatiguing contractions, the signal might be considered stationary for epoch durations of the order of about $1-2$ seconds [1]. In addition, no overlap was performed between consecutive epochs. Such a choice was suggested by Farina et al. [21] which states that overlaps do not provide significant benefits.

2) ECG processing: ECG signals were digitally filtered via a zero-phase Butterworth IIR band-pass filter $(1-40 \mathrm{~Hz})$ and an IIR comb notch filter $(50 \mathrm{~Hz})$. Then, the well known Pan-Tompkins method [22] was used to detect R-peaks. At the end, a regularly sampled HRV series was obtained via a spline interpolation, fixing the sampling frequency equal to 4 Hz. A set of features in the time and frequency domain was estimated from every HRV series. Specifically, they were: mean RR (meanRR), standard deviation of RR (stdRR), the square root of the mean squared differences of successive NN intervals (RMSSD), the number of interval differences of successive NN intervals greater than $50 \mathrm{~ms}$ (NN50), the proportion derived by dividing NN50 by the total number of $\mathrm{NN}$ intervals (pNN50), the HRV triangular index (HRVtri), and the triangular interpolation of $\mathrm{NN}$ interval histogram (TINN), the power spectrum estimated at low frequency (LF), at high frequency (HF), their ratio (LF/HF), and finally the standard deviations (SD1 and SD2) estimated along the two axes of the ellipse in the Poincare plot [23]. Frequency features were estimated via the autoregressive model (AR).

\section{Statistical analysis}

Statistical analysis was performed to investigate potential statistically significant differences in HRV-features related to muscle fatigue. To this aim, subjects were divided in two groups according to their MNF and MDF trends during the task. In fact, subjects showing decreasing trends were grouped and considered as fatigued $(F)$. On the contrary, the remaining subjects, showing non-decreasing trends, were grouped and considered as non-fatigued $(N-F)$. In this frame, a Mann-Whitney U-test was used to compare the features belonging to the two different groups. Of note, the corresponding resting phase value was subtracted from every feature $\left(\right.$ Feat $=$ Feat $_{\text {Task }}-$ Feat $\left._{\text {Rest }}\right)$.

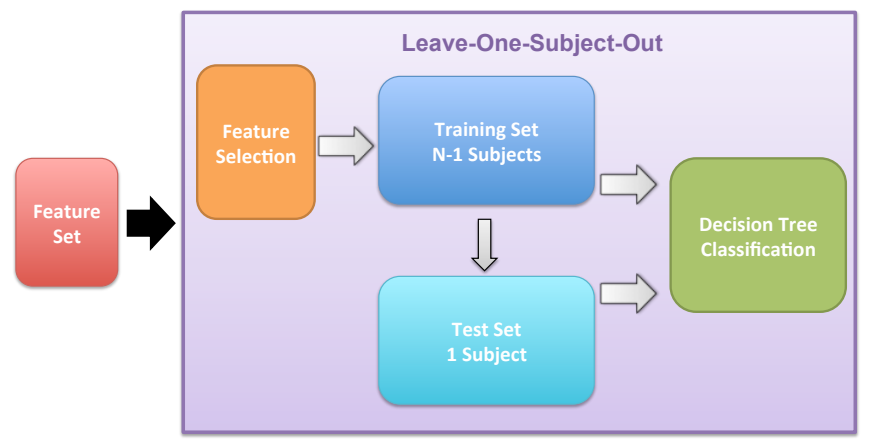

Fig. 2. Scheme of the proposed Leave-One-Subject-Out architecture (LOSO) architecture.

\section{Pattern recognition}

A Leave-One-Subject-Out architecture (LOSO) was applied to recognize subjects belonging to the two different groups, i.e. fatigued and non-fatigued, using a Decision Tree (DT) classifier and a nested feature selection. Only the features showing a statistical significant difference between the two groups were used. The DT classifier was iteratively trained on $N-1$ subjects, and then tested on the remaining 1 subject. The algorithm of feature selection consisted in selecting only the features showing, during the training test, 
TABLE I
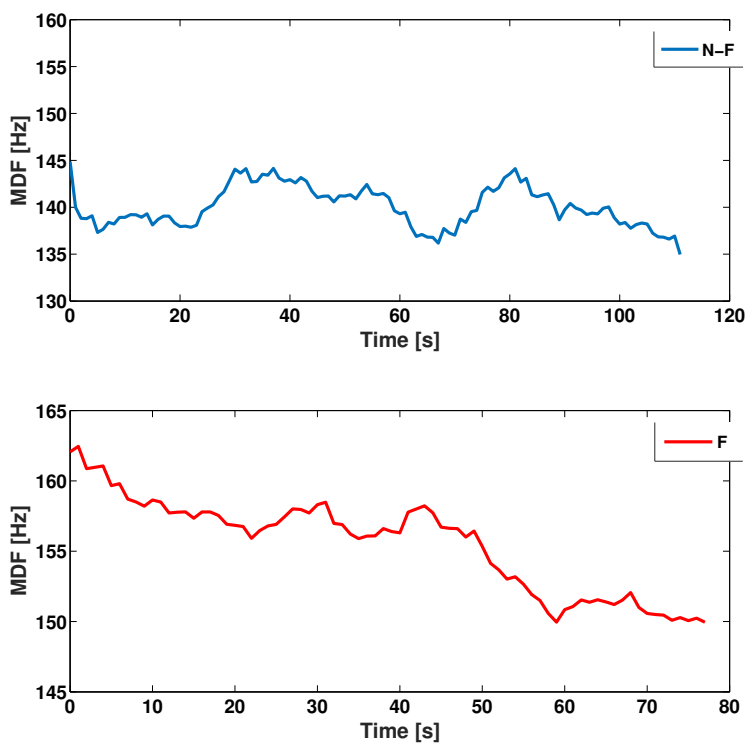

Fig. 3. Example of MDF trends. In red it is reported a decreasing MDF trend related to a subject belonging to the F group. Differently, in blue it is reported an example of a subject who did not experience the fatigue condition.

a correlation ratio higher than a threshold. Such a threshold was set as the median value of the correlation ratios estimated in that specific iteration. A confusion matrix was returned by the proposed algorithm as a result.

\section{RESULTS}

According to the MNF and MDF trends, 17 subjects ( 9 females and 8 males) experienced muscle fatigue ( $\mathrm{F}$ group), while the remaining 15 subjects ( 7 females and 8 males) did not. Figure 3 shows two MDF curves over time relative to a fatigued subject (upper) compared to nonfatigued subject (lower) A decreasing MDF trend, indicating fatigue condition, is reported in red, while in blue it is shown a MDF trend related to a subject who did not experience the fatigue condition.

The results concerning the statistical analysis between the features estimated from the subjects belonging to the fatigued and non-fatigued groups are reported in Table I. Significant results ( $\mathrm{p}$-value $<0.05)$ are highlighted in bold, while an arrow indicates the lower $(\downarrow)$ or higher $(\uparrow)$ feature value in the fatigue $(F)$ state. More specifically, a significant lower feature value was observed in $F$-group by analyzing RMSSD, NN50, HF and SD1. Differently, a higher LF value was observed in $F$-group.

In Table II the confusion matrix concerning the proposed LOSO algorithm is reported. The elements on the main diagonal report the percentage of correct recognition, while the other elements report the percentage of incorrect detection. An overall accuracy equal to $78.24 \%$ was achieved.

\section{DISCUSSION AND CONCLUSION}

In this study HRV-features were analyzed in relation to muscle fatigue. In the literature, muscular activity has
IN TABLE THE P-VALUES CORRESPONDING TO THE MANN-WHITNEY U-TEST ARE REPORTED. THE SIGNIFICANT RESULTS (P-VALUE < 0.05) ARE HIGHLIGHTED IN BOLD. AN ARROW INDICATES A DECREASE $(\downarrow)$ OR INCREASE $(\uparrow)$ OF THE MEDIAN VALUE IN THE FATIGUED $(F)$ STATE AS COMPARED TO THE NON-FATIGUED STATE.

\begin{tabular}{|c|c|c|}
\hline \multicolumn{3}{|c|}{ N-F vs F } \\
\hline Features & p-value & Trend \\
\hline meanRR & $6.47 \mathrm{E}-02$ & \\
\hline stdRR & $1.16 \mathrm{E}-01$ & \\
\hline RMSSD & $\mathbf{1 . 2 7 E - 0 2}$ & $\downarrow$ \\
\hline NN50 & $\mathbf{4 . 4 4 E - 0 2}$ & $\downarrow$ \\
\hline pNN50 & $7.31 \mathrm{E}-02$ & \\
\hline HRVtri & $9.81 \mathrm{E}-02$ & \\
\hline TINN & $1.69 \mathrm{E}-01$ & \\
\hline LF & $\mathbf{4 . 4 4 E - 0 2}$ & $\uparrow$ \\
\hline HF & $\mathbf{4 . 4 4 E - 0 2}$ & $\downarrow$ \\
\hline LF/HF & $9.89 \mathrm{E}-01$ & \\
\hline SD1 & $\mathbf{1 . 2 7 E - 0 2}$ & $\downarrow$ \\
\hline SD2 & $1.27 \mathrm{E}-02$ & \\
\hline
\end{tabular}

TABLE II

CONFUSION MATRIX RELATED TO THE PROPOSED LOSO ALGORITHM (AVERAGE ACCURACY EQUAL TO 78.23\% ).

\begin{tabular}{|c|c|c|c|}
\hline \multicolumn{2}{|c|}{} & \multicolumn{2}{c|}{ Predicted } \\
\cline { 3 - 4 } \multicolumn{2}{|c|}{} & N-F & F \\
\hline \multirow{2}{*}{ Actual } & N-F & $\mathbf{8 0 . 0 0 \%}$ & $20.00 \%$ \\
\cline { 2 - 4 } & F & $23.53 \%$ & $\mathbf{7 6 . 4 7 \%}$ \\
\hline
\end{tabular}

been usually investigated by studying post-fatigue or overtraining states caused by exercises at increasing intensities or aerobic exercises. In this study, 32 healthy subjects were enrolled to evaluate HRV-features during fatiguing exercises, i.e. a prolonged isometric contraction of the biceps brachii long head muscle. Subjects performed an isometric exercise lifting a load equal to $40 \%$ of their MVC to a horizontal position and maintaining it until it became impossible to keep the horizontal position. Median and mean frequency was estimated from the spectrum of the sEMG signals and were used as reference parameters to detect fatigued subjects.

HRV features were extracted in the time, frequency and nonlinear domains. A statistical analysis and a pattern recognition was applied on HRV feature-set to characterize and distinguish fatigued subjects from the non-fatigued ones. Results of the statistical analysis showed relevant HRVfeatures, in all domains, able to significantly characterize subjects according to their actual muscular conditions. More specifically, time-domain features showed significant reduced RMSSD and NN50 values in the fatigued group. This might indicate an increasing heart rate in presence of fatigue. Moreover, frequency-domain features showed an increased LF, and a reduced HF in fatigued subjects. Such a condition is usually detected in stressed people [24]. Furthermore, a study of HRV non-linear features revealed that a lower SD1 value can be detected in subjects experiencing muscle fatigue.

The significant features were also used as input of a pattern recognition system. Classification results revealed that the proposed algorithm was able to correctly recognize muscular conditions by means of HRV-features with an 
overall accuracy of $78.24 \%$.

In conclusion, these results confirm that perceived exertion might not be related to the actual muscle-fatigue-state of the subjects. Indeed, nearly half of the subjects interrupted the exercise before the arise of the real state of muscle fatigue. Our preliminary results suggest that real-time monitoring of HRV during exercises might provide an important help in detecting actual muscle states. In addition, according to the study of Mehta and Agnew [25], mental workload adversely affects physical capacity. Since HRV is commonly used to investigate emotional and psychological states (e.g., [26][29] and references therein), as well as stress conditions (e.g., [30] and references therein), its study might provide a description of both phenomena, i.e. muscle fatigue and mental state.

A possible future application might be related to the development of novel prosthesis. In fact, criteria based on these phenomena might help researcher in evaluating both stress and fatigue in residual muscles during the testing phases [31] in a non-invasive way.

Future works will be focused on the study of the temporal evolution of HRV-related features during fatiguing exercise. Gender differences will be also investigated to better clarify the gender effect on the relationship between HRV and sEMG.

\section{REFERENCES}

[1] R. Merletti and P. A. Parker, Electromyography: physiology, engineering, and non-invasive applications. John Wiley \&amp; Sons, 2004, vol. 11.

[2] H. Piper, "Elektrophysiologie menschlicher muskeln springer verlag," 1912.

[3] D. B. Chaffin, "Localized muscle fatigue-definition and measurement." Journal of Occupational and Environmental Medicine, vol. 15, no. 4, pp. 346-354, 1973.

[4] C. De Luca, "Myoelectric manifestation of localized muscular fatigue in humans," in IEEE Transactions On Biomedical Engineering, vol. 30, no. 8. IEEE-INST Electrical Electronics Engineers Inc 445 Hoes Lane, Piscataway, NJ 08855 USA, 1983, pp. 531-531.

[5] W. Li and K. Sakamoto, "The influence of location of electrode on muscle fiber conduction velocity and emg power spectrum during voluntary isometric contraction measured with surface array electrodes," Applied Human Science, vol. 15, no. 1, pp. 25-32, 2001.

[6] L. Lindstrom, "Muscular fatigue and action potential conduction velocity changes studied with frequency analysis of emg signals." Electromyogr Clin Neurophysiol, vol. 10, pp. 341-356, 1970.

[7] A. Phinyomark, C. Limsakul, H. Hu, P. Phukpattaranont, and S. Thongpanja, The usefulness of mean and median frequencies in electromyography analysis. INTECH Open Access Publisher, 2012.

[8] R. Lepers, C. Hausswirth, N. Maffiuletti, J. Brisswalter, and J. Van Hoecke, "Evidence of neuromuscular fatigue after prolonged cycling exercise," Medicine and science in sports and exercise, vol. 32, no. 11, pp. 1880-1886, 2000.

[9] L. Sheldahl, L. Wann, P. Clifford, F. Tristani, L. Wolf, and J. Kalbfleisch, "Effect of central hypervolemia on cardiac performance during exercise," Journal of Applied Physiology, vol. 57, no. 6, pp. 1662-1667, 1984.

[10] J. A. Schwane, S. R. Johnson, C. B. Vandenakker, and R. B. Armstrong, "Delayed-onset muscular soreness and plasma cpk and ldh activities after downhill running." Medicine and science in sports and exercise, vol. 15, no. 1, pp. 51-56, 1982.

[11] T. L. Hooper, D. M. Dunn, J. E. Props, B. A. Bruce, S. F. Sawyer, and J. A. Daniel, "The effects of graded forward and backward walking on heart rate and oxygen consumption," Journal of Orthopaedic \&amp; Sports Physical Therapy, vol. 34, no. 2, pp. 65-71, 2004.

[12] A. S. Oliveira and M. Gonçalves, "Positioning during resistance elbow flexor exercise affects electromyographic activity, heart rate, and perceived exertion," The Journal of Strength \&amp; Conditioning Research, vol. 23, no. 3, pp. 854-862, 2009.
[13] C. J. Mottram, J. M. Jakobi, J. G. Semmler, and R. M. Enoka, "Motorunit activity differs with load type during a fatiguing contraction," Journal of neurophysiology, vol. 93, no. 3, pp. 1381-1392, 2005.

[14] B. Makivić, M. Djordjević Nikić, and M. S. Willis, "Heart rate variability (hrv) as a tool for diagnostic and monitoring performance in sport and physical activities," Journal of Exercise Physiologyonline, vol. 16, no. 3, pp. 100-109, 2013.

[15] C. Scott, F. Wyatt, J. Winchester, K. Williamson, A. Welter, and S. Brown, "Physiological breakpoints and maximal steady-state of cycling," Journal of Exercise Physiology Online, vol. 18, no. 3, pp. 33-46, 2015.

[16] J.-L. Chen, D.-P. Yeh, J.-P. Lee, C.-Y. Chen, C.-Y. Huang, S.-D. Lee, C.-C. Chen, T. B. Kuo, C.-L. Kao, and C.-H. Kuo, "Parasympathetic nervous activity mirrors recovery status in weightlifting performance after training," The Journal of Strength \& Conditioning Research, vol. 25, no. 6, pp. 1546-1552, 2011.

[17] R. Mehta, "Impacts of obesity and stress on neuromuscular fatigue development and associated heart rate variability," International Journal of Obesity, vol. 39, no. 2, pp. 208-213, 2015.

[18] T. Kiryu, T. Abe, T. Gocho, Y. Ushiyama, and M. Okada, "Snapshot evaluation of fatigue during skiing exercise," in Engineering in Medicine and Biology Society, 1998. Proceedings of the 20th Annual International Conference of the IEEE, vol. 5. IEEE, 1998, pp. 27752778.

[19] J. S. Petrofsky and M. Laymon, "Blood pressure and heart rate responses during a fatiguing isometric exercise in paraplegic men with hypertension," European journal of applied physiology, vol. 83, no. 4, pp. 274-282, 2000.

[20] S. A. Zaman, D. T. MacIsaac, and P. A. Parker, "Repeatability of surface emg-based single parameter muscle fatigue assessment strategies in static and cyclic contractions," in Engineering in Medicine and Biology Society, EMBC, 2011 Annual International Conference of the IEEE. IEEE, 2011, pp. 3857-3860.

[21] D. Farina and R. Merletti, "Comparison of algorithms for estimation of emg variables during voluntary isometric contractions," Journal of Electromyography and Kinesiology, vol. 10, no. 5, pp. 337-349, 2000.

[22] J. Pan and W. J. Tompkins, "A real-time qrs detection algorithm," IEEE transactions on biomedical engineering, no. 3, pp. 230-236, 1985.

[23] T. F. of the European Society of Cardiology et al., "Heart rate variability standards of measurement, physiological interpretation, and clinical use," Eur heart J, vol. 17, pp. 354-381, 1996.

[24] D. McDuff, S. Gontarek, and R. Picard, "Remote measurement of cognitive stress via heart rate variability," in Engineering in Medicine and Biology Society (EMBC), 2014 36th Annual International Conference of the IEEE. IEEE, 2014, pp. 2957-2960.

[25] R. K. Mehta and M. J. Agnew, "Influence of mental workload on muscle endurance, fatigue, and recovery during intermittent static work," European journal of applied physiology, vol. 112, no. 8, pp. 2891-2902, 2012.

[26] R. A. Calvo and S. D’Mello, "Affect detection: An interdisciplinary review of models, methods, and their applications," IEEE Transactions on affective computing, vol. 1, no. 1, pp. 18-37, 2010.

[27] M. Nardelli, G. Valenza, A. Greco, A. Lanata, and E. P. Scilingo, "Recognizing emotions induced by affective sounds through heart rate variability," IEEE Transactions on Affective Computing, vol. 6, no. 4, pp. 385-394, 2015.

[28] G. Valenza, A. Greco, L. Citi, M. Bianchi, R. Barbieri, and E. Scilingo, "Inhomogeneous point-processes to instantaneously assess affective haptic perception through heartbeat dynamics information," Scientific Reports, vol. 6, 2016.

[29] G. Valenza, A. Greco, C. Gentili, A. Lanata, L. Sebastiani, D. Menicucci, A. Gemignani, and E. Scilingo, "Combining electroencephalographic activity and instantaneous heart rate for assessing brain-heart dynamics during visual emotional elicitation in healthy subjects," Phil. Trans. R. Soc. A, vol. 374, no. 2067, p. 20150176 , 2016.

[30] A. Lanatà, G. Valenza, A. Greco, C. Gentili, R. Bartolozzi, F. Bucchi, F. Frendo, and E. P. Scilingo, "How the autonomic nervous system and driving style change with incremental stressing conditions during simulated driving," IEEE Transactions on Intelligent Transportation Systems, vol. 16, no. 3, pp. 1505-1517, 2015.

[31] C. Cipriani, F. Zaccone, S. Micera, and M. C. Carrozza, "On the shared control of an emg-controlled prosthetic hand: analysis of userprosthesis interaction," IEEE Transactions on Robotics, vol. 24, no. 1, pp. 170-184, 2008. 